Tropical Journal of Pharmaceutical Research August 2020; 19 (8): 1591-1597

ISSN: $1596-5996$ (print); 1596-9827 (electronic)

(C) Pharmacotherapy Group, Faculty of Pharmacy, University of Benin, Benin City, 300001 Nigeria

Available online at http://www.tjpr.org

Original Research Article

http://dx.doi.org/10.4314/tjpr.v19i8.4

\title{
Inhibition of miR-665 alleviates neuropathic pain by targeting SOCS1
}

\author{
Yongqiang Lin ${ }^{1}$, Mengjia Li ${ }^{2 *}$, Gaofeng Rao ${ }^{1}$, Wenfu Zhang ${ }^{1}$, Xuyan Chen ${ }^{3}$ \\ ${ }^{1}$ Department of Rehabilitation Medicine, ${ }^{2}$ Department of Medical Laboratory Science, The First People's Hospital of Wenling, \\ The Affiliated Wenling Hospital of Wenzhou Medical University, Taizhou City, Zhejiang Province 317500, ${ }^{3}$ Department of \\ Physical Medicine \& Rehabilitation, The Second Affiliated Hospital \& Yuying Children's Hospital of Wenzhou Medical University, \\ Wenzhou City, Zhejiang Province 325000, China
}

*For correspondence: Email: QOI5cx@163.com; Tel: +86-576-89668210

Sent for review: 2 June 2020

Revised accepted: 3 August 2020

\begin{abstract}
Purpose: To investigate the effect of miR-665 in neuropathic pain and the possible molecular mechanism involved.

Methods: A neuropathic pain model was established using chronic constriction injury (CCI) methods in Sprague Dawley (SD) rats. Mechanical and thermal hyperalgesia were measured using paw withdrawal threshold (PWT) and paw withdrawal latency $(P W L)$, respectively. The inflammation response was determined by assessing the production of inflammation factors. The target relationship of miR-665 and suppressor of cytokine signaling 1 (SOCS1) was verified by luciferase assay.

Results: In the CCl rat model, PWT and PWL decreased following treatment with miR-665 $(p<0.01)$. MiR-665 was elevated in the spinal cord and microglia of $\mathrm{CCl}$ rats at different time points $(p<0.01)$. Down-regulation of miR-665 increased PWT and PWL and inhibited the production of interleukin (IL)-1 $\beta$, IL-6, and tumor necrosis factor (TNF)- $\alpha$ in CCI rats $(p<0.01)$. Luciferase assay results indicate that SOCS1 was the target of miR-665 ( $p<0.01)$. SOCS1 decreased in CCl rats $(p<0.01)$ after treatment with miR-665. MiR-665 negatively regulated the expression of SOCS1 $(p<0.01)$. Down-regulation of SOCS1 reversed the alleviating effect of decreased miR-665 on pain sensitivity and inflammation response $(p<0.01)$.

Conclusion: Down-regulation of miR-665 alleviates neuropathic pain by targeting SOCS1, and hence making miR-665 a promising therapeutic target for neuropathic pain.
\end{abstract}

Keywords: MiR-665, SOCS1, Neuropathic pain, CCI, Spinal cord

\begin{abstract}
This is an Open Access article that uses a fund-ing model which does not charge readers or their institutions for access and distributed under the terms of the Creative Commons Attribution License (http://creativecommons.org/licenses/by/4.0) and the Budapest Open Access Initiative (http://www.budapestopenaccessinitiative.org/read), which permit unrestricted use, distribution, and reproduction in any medium, provided the original work is properly credited.

Tropical Journal of Pharmaceutical Research is indexed by Science Citation Index (SciSearch), Scopus, International Pharmaceutical Abstract, Chemical Abstracts, Embase, Index Copernicus, EBSCO, African Index Medicus, JournalSeek, Journal Citation Reports/Science Edition, Directory of Open Access Journals (DOAJ), African Journal Online, Bioline International, Open-J-Gate and Pharmacy Abstracts
\end{abstract}

\section{INTRODUCTION}

Chronic neuropathic pain is long-lasting pathological pain triggered by a lesion or disease of the somatosensory system [1]. Neuropathic pain is characterized by ectopic pain without pain stimulation and a hyperalgesia response to harmful stimulation caused by nervous system injury or dysfunction [2]. At present, neuropathic pain is a serious threat to public health that affects nearly $8 \%$ of adults [3]. Furthermore, neuropathic pain is difficult to manage and poses a challenge to quality of life for patients, especially because traditional drug therapy is 
ineffective [3]. Thus, it is essential to explicate the molecular mechanism of neuropathic pain to develop effective therapeutic strategies.

Small non-coding microRNAs (miRNAs) ( 22 nucleotides) serve as a post-transcriptional regulator in many diseases via inhibiting the expression of target genes [4]. Accumulating evidence shows that miRNAs participate in the regulation of neuropathic pain [5-7]. For example, the down-regulated miR-144 in a neuropathic pain mouse model modulates pain sensitivity and the inflammation response through targeting RAS P21 protein activator 1 (RASA1) [8]. Elevated miR-206 attenuates chronic constriction injury (CCl)-induced neuropathic pain through directly binding to brain-derived neurotrophic factor (BDNF) and activates the MEK/extracellular signal-regulated kinase (ERK) pathway [6].

miR-150 suppresses mechanical and thermal hyperalgesia by targeting AKT serine/threonine kinase 3 (AKT3) [7]. In particular, miR-665 controls inflammatory responses and cell apoptosis. miR-665 overexpression promotes cell apoptosis and colitis in inflammatory bowel disease through suppressing the expression of X-box-binding protein-1 (XBP1) and ORMDL sphingolipid biosynthesis regulator 3 (ORMDL3) [9]. Moreover, miR-665 exerts a neuroprotective effect in a rat model with sevoflurane-induced cognitive dysfunction through targeting insulinlike growth factor 2 and activating the phosphoinositide 3-kinase $(\mathrm{PI} \mathrm{K}) /$ protein kinase $B(A k t)$ pathway [10]. However, the effect of miR665 on chronic neuropathic pain remains unclear. Therefore, the role of miR-665 in neuropathic pain was investigated in the current study.

In this study, the influences and potential modulation mechanism of miR-665 on hyperalgesia and inflammation response in neuropathic pain induced by $\mathrm{CCl}$ were studied.

\section{EXPERIMENTAL}

\section{Establishment of chronic constriction injury (CCI) model}

The neuropathic pain rat model was established using $\mathrm{CCl}$ methods. Sprague Dawley (SD) rats weighing $250 \pm 20 \mathrm{~g}$ were obtained from Beijing Laboratory Animal Research Center (Beijing, China) and randomly grouped into sham and $\mathrm{CCl}$ groups. For establishing the $\mathrm{CCl}$ model, chloral hydrate $(350 \mathrm{mg} / \mathrm{kg})$ was used to anesthetize the rats via intraperitoneal injection. In the $\mathrm{CCl}$ group, the left sciatic nerves were exposed at the mid-thigh level and were ligated with chromic catgut at 4 sites [11]. The interval between the two ligations was approximately $1 \mathrm{~mm}$. The left sciatic nerves in the sham group were exposed to the middle thigh without ligation. At indicated times after ligation, the L4-L6 spinal cord of rats were collected for subsequent studies. The spinal microglia were isolated using $70 \%$ isotonic Percoll solution to perform follow-up experiments. To investigate the effect of mi-665 on pain sensitivity and the production of inflammation factors and to determine the underlying mechanism, the rats in the sham and $\mathrm{CCl}$ groups were injected negative control antagomir (NC antagomir), miR-665 antagomir, or shRNA adenovirus plasmid of suppressor of cytokine signaling 1 (SOCS1) (GenePharma, Shanghai, China) in the medullary sheath. The animal experiments in the study were performed according to the National Institutes of Health guidelines for the use of experimental animals [12]. The study was approved by the Ethics Committee of The First People's Hospital of Wenling. The Affiliated Wenling Hospital of Wenzhou Medical University (approval no. 2017042) approved the study.

\section{Pain threshold assessment}

Mechanical ectopic pain was assessed by determining the paw withdrawal threshold (PWT) under the von Frey hair test according to methods used in previous studies $[6,13]$. The rats were placed in a transparent plastic box with a metal mesh floor. To determine the PWT, the pelma was pressed from below via the mesh floor with an electronic von Frey filament. The force applied when each rat withdrew their paw was recorded The PWT result was expressed as the mean of at least three trials. According to the Hargreaves method, the paw withdrawal latency (PWL) was measured by evaluating the thermal sensitivity of rats under thermal radiation. The rats were put in a Plexiglas box on a high glass table. The source of radiation was concentrated in the center of the plantar surface of each rear paw underneath the glass table [11]. The heat intensity was set to produce a PWL of approximately $10 \mathrm{~s}$ in normal animals. To avoid tissue injury, the cut-off time was set at $20 \mathrm{sec}$. The rats underwent three rounds of thermal stimulation every 5 to $10 \mathrm{~min}$.

\section{Quantitative real-time polymerase chain reaction (qRT-PCR)}

The extraction of an RNA sample from the spinal cord and spinal microglia was conducted using QIAsymphony RNA Kit (QIAGEN, Hilden, Germany). The isolated RNA was used to 
produce cDNA through reverse transcription reactions using the ReadyScript cDNA Synthesis Mix (Sigma, St. Louis, MO, USA) or miRNA cDNA Synthesis Kit (ComWin Biotech, Beijing, China). Quantitative PCR (qPCR) reactions were then performed with the generated CDNA as templates using SYBR Green PCR Master Mix (Thermo Fisher Scientific, Waltham, MA, USA) or miRNA qPCR Assay Kit (ComWin Biotech, Beijing, China). The primer sequences used for PCR are listed in Table $1[14,15]$.

\section{Enzyme-linked immunosorbent assay (ELISA)}

Rat spinal cords were collected to determine the levels of IL-1 $\beta$, IL- 6 , and TNF- $\alpha$ (Abcam, Cambridge, UK) using ELISA assay kits following the manufacturers' protocols.

\section{Luciferase assay}

The pmiRGLO vector (Promega, Madison, WI, USA) containing wild and mutant 3'-untranslated region (UTR) of SOCS1 were designed and cloned. The pmiRGLO-SOCS1-WT, pmiRGLOSOCS1-MUT, miR-665 mimics, and negative control mimics (NC mimics) (GenePharma, Shanghai, China) were co-transfected into HEK293 cells using Lipofectamine 2000 (Invitrogen, Carlsbad, CA, USA). The HEK293 cells were collected at $48 \mathrm{~h}$ post-transfection to measure the relative luciferase activities via standardizing to the Renilla luciferase activity.

\section{Western blot}

The spinal cord was lysed in RIPA buffer to isolate total proteins, which were quantified using the bicinchoninic acid (BCA) method (ComWin Biotech, Beijing, China). Subsequently, $40 \mu \mathrm{g}$ lysate was separated on an SDS-PAGE gel. The lysates were then transferred to a PVDF membrane. Next, the membrane was blocked with $4 \%$ non-fat milk. The membrane was probed with anti-SOCS1 antibody (1:500) (Abcam, Cambridge, UK) and anti- $\beta$-actin antibody (1:1000) (Abcam, Cambridge, UK) at $4{ }^{\circ} \mathrm{C}$ for 12 $\mathrm{h}$ and incubated with HRP-labeled anti-lgG secondary antibody (Abcam, Cambridge, UK) at $37{ }^{\circ} \mathrm{C}$ for $1 \mathrm{~h}$. $\beta$-Actin was used as a control protein. Protein bands were visualized using the
ECL Western blot kit (Thermo Fisher Scientific, Waltham, MA, USA) and quantified using Image $\mathrm{J}$ software.

\section{Statistical analysis}

SPSS version 22.0 (Chicago, IL, USA) was used for data analysis. All data are presented as mean \pm standard deviation (SD). The difference between two groups was determined using the Student's $t$-test. One-way ANOVA was utilized to evaluate differences among multiple groups. $P<$ 0.05 was regarded as statistically significant.

\section{RESULTS}

\section{MiR-665 expression was elevated in dorsal root ganglion of $\mathrm{CCl}$ rats}

After the establishment of $\mathrm{CCl}$ rats, the PWT and PWL were decreased in the $\mathrm{CCl}$ rats compared to the sham rats $(p<0.01$, Figure $1 \mathrm{~A}$ and $\mathrm{B})$. The results revealed that $\mathrm{CCl}$ rats had increased mechanical and thermal hyperalgesia and that the $\mathrm{CCl}$ model was successful. The results also showed miR-665 expression was elevated in the spinal cord and microglia of $\mathrm{CCl}$ rats in contrast to the sham group ( $p<0.01$, Figure $1 \mathrm{C}$ and D). Therefore, the expression of miR-665 was increased in the dorsal root ganglion of $\mathrm{CCl}$ induced neuropathic pain rats.

\section{Down-regulation of miR-665 alleviated pain sensitivity of $\mathrm{CCI}$ rats}

To elucidate the influence of aberrantly expressed miR-665 in neuropathic pain, the rats were injected with miR-665 antagomir or NC antagomir to knockdown miR-665. The decreased levels of miR-665 in sham and $\mathrm{CCl}$ rats injected with miR-665 antagomir were verified by $q R T-P C R$ assay $(p<0.01$, Figure 2 A). The miR-665 antagomir in $\mathrm{CCl}$ rats considerably increased the PWT and PWL compared to $\mathrm{CCl}$ rats injected with $\mathrm{NC}$ antagomir $(p<0.01)$, while there was no significant difference between the sham rats injected with NC antagomir and miR-665 antagomir (Figure 2 $B)$. Thus, the down-regulation of miR-665 alleviated pain sensitivity of $\mathrm{CCl}$ rats.

Table 1: PCR primer sequences

\begin{tabular}{|c|c|c|}
\hline Gene & Forward & Reverse \\
\hline MiR-665 & 5'-AACAAGACCAGGAGGCTGAGG-3' & 5'-CAGTGCAGGGTCCGAGGT-3' \\
\hline IL-1 $\beta$ & 5'-CATCTTTGAAGAAGAGCCCG-3' & 5'-TGTTCGTTGCTGTTTTAGGG-3' \\
\hline IL-6 & 5'-CGAAAGTCAACTCCATCTGCC-3' & 5'--GGCAACTGGCTGGAAGTCTCT-3' \\
\hline TNF- $\alpha$ & 5'-CCAGGAGAAAGTCAGCСTCCT-3' & 5'-TCATACCAGGGCTTGAGCTCA-3' \\
\hline
\end{tabular}


A

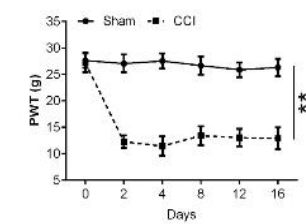

C

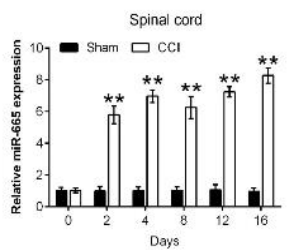

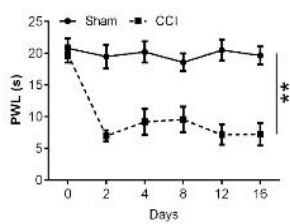

D

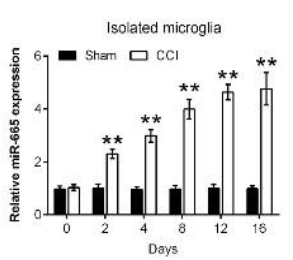

Figure 1: MiR-665 was overexpressed in the dorsal root ganglion of $\mathrm{CCl}$ rats. A. PWT was measured to reflect the mechanical hyperalgesia of $\mathrm{CCl}$ rats. $\mathrm{B}$. PWL was determined to indicate thermal hyperalgesia of $\mathrm{CCl}$ rats. C. miR-665 expression in the spinal cord of $\mathrm{CCl}$ rats determined using qRT-PCR assay. D. miR665 expression in microglia of $\mathrm{CCl}$ rats determined using qRT-PCR; ${ }^{* *} p<0.01$

A

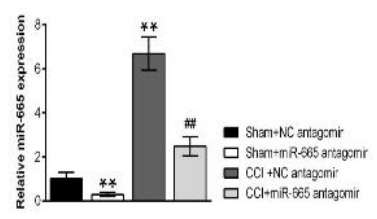

B
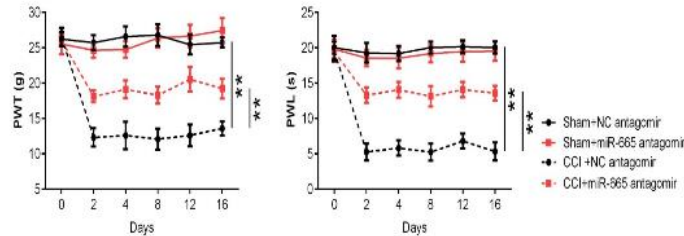

Figure 2: Down-regulation of miR-665 alleviated pain sensitivity of $\mathrm{CCl}$ rats. A. qRT-PCR assay determined the expression of miR-665 in $\mathrm{CCl}$ rats injected with miR-665 antagomir or NC antagomir. B. The PWT and PWL were determined in $\mathrm{CCl}$ rats injected with miR665 antagomir or NC antagomir; ${ }^{* *} p<0.01$. \#\#p < 0.01

Down-regulation of miR-665 inhibited the production of inflammation factors in $\mathrm{CCl}$ rat spinal cord

The levels of IL-1 $\beta, I L-6$, and TNF- $\alpha$ were increased in $\mathrm{CCl}$ rats injected with $\mathrm{NC}$ antagomir compared to sham rats injected with $\mathrm{NC}$ antagomir ( $p<0.01$, Figure 3 A and B). Nevertheless, miR-665 antagomir significantly decreased IL-1 $\beta$, IL-6, and TNF- $\alpha$ levels in $\mathrm{CCl}$ rats compared to $\mathrm{CCl}$ rats injected with $\mathrm{NC}$ antagomir ( $p<0.01$, Figure $3 \mathrm{~A}$ and B). Thus,

down-regulation of miR-665 reduced the production of inflammation factors in $\mathrm{CCl}$ rats.

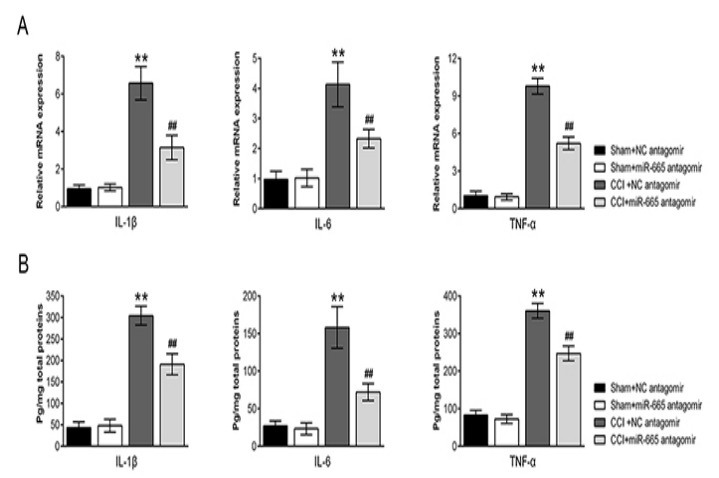

Figure 3: Down-regulation of miR-665 reduced production of inflammation factors in the spinal cord of $\mathrm{CCl}$ rats. A. mRNA levels of IL-1 $\beta, \mathrm{IL}-6$, and TNF- $\alpha$ in the spinal cord of $\mathrm{CCl}$ rats injected with miR-665 antagomir or NC antagomir were measured using qRT-PCR. B. The protein levels of IL-1 $1 \beta, \mathrm{IL}-6$, and TNF- $\alpha$ in spinal cord $\mathrm{CCl}$ rats injected with miR-665 antagomir or NC antagomir were measured by ELISA; ${ }^{* *} p<0.01 . \# \# p<0.01$

\section{SOCS1 was the target gene of miR-665}

TargetScan (http//www.targetscan.org) was screened to find the potential target gene of miR665. Results showed that SOCS1 might be a target of miR-665 and the putative binding sites of miR-665 and SOCS1 are exhibited in Figure 4 A. Luciferase assay results indicated that miR665 mimic markedly decreased the luciferase activity in HEK293 cells transfected with the wild type 3'-UTR of SOCS1 ( $p<0.01$, Figure $4 \mathrm{~B}$ ). However, luciferase activity in HEK293 cells transfected with the mutant 3'-UTR of SOCS1 had no significant differences between the miR665 and NC mimic groups (Figure 4 B). Western blot results showed that SOCS1 levels in $\mathrm{CCl}$ rats were significantly reduced compared to the sham group (all $p<0.01$, Figure 4 C). Furthermore, the decreased levels of SOCS1 in $\mathrm{CCl}$ rats were increased by miR-665 antagomir (all $p<0.01$, Figure 4 D). Therefore, SOCS1 was the target gene of miR-665 and miR-665 negatively regulated the expression of SOCS1.

\section{MiR-665 aggravated neuropathic pain via regulation of SOCS1 expression}

To determine the regulation mechanism of $\mathrm{miR}$ 665 on neuropathic pain, the $\mathrm{CCl}$ rats were injected with miR-665 antagomir and adenovirus plasmid of shSOCS1. Results showed that miR665 antagomir increased the protein level of SOCS1 $(p<0.01)$, while shSOCS1 restored 
SOCS1 levels $(p<0.01$, Figure 5 A). miR-665 antagomir significantly increased the PWT and PWL, but shSOCS1 decreased the PWT and PWL (all $p<0.01$, Figure $5 \mathrm{~B}$ ). Moreover, the inhibitory effects of miR-665 down-regulation on the levels of IL-1 $\beta, I L-6$, and TNF- $\alpha$ were suppressed by down-regulation of SOCS1 (all $p$ $<0.01$, Figure $5 \mathrm{C}$ ). Thus, miR-665 aggravated neuropathic pain through targeting SOCS1.

\section{DISCUSSION}

Neuropathic pain is a pathological and longlasting pain that affects approximately $8 \%$ of adults [3]. Neuropathic pain is a serious threat to public health and it reduces quality of life for patients [3]. Therefore, it is crucial to investigate the molecular mechanism of neuropathic pain to find effective therapeutic strategies.

miRNA is a post-transcriptional regulator in many diseases, including neuropathic pain [16]. miR665 acts as a neuroprotective regulator and modulates inflammatory responses $[9,10]$. Thus, miR-665 may regulate neuropathic pain. To elucidate the roles of miR-665 in neuropathic pain regulation, a neuropathic pain model was established using the CCl method. The PWT and PWL were used as indices of mechanical and thermal hyperalgesia [17].
A

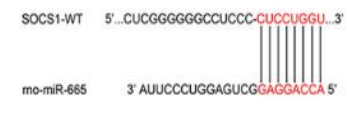

SOCS1-NUT S. CUCGGGGGGCCUCCC.GAGGACCA.3.3

C

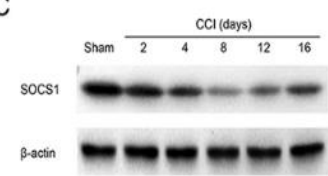

D

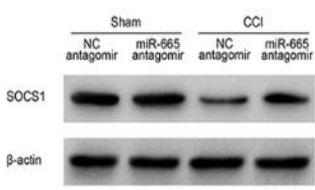

B
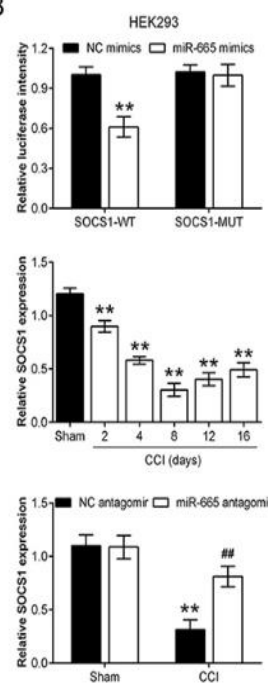

Figure 4: SOCS1 is the target gene of miR-665. A. The putative binding site of miR-665 and SOCS1. B. Luciferase assay was used to verify the relationship between miR-665 and SOCS1. C. The protein levels of SOCS1 in $\mathrm{CCl}$ rats determined using Western blot. D. The protein levels of SOCS1 in $\mathrm{CCl}$ rats injected with miR-665 antagomir or NC antagomir were measured using Western blot; ${ }^{* *} p<0.01$. \#\#p $<0.01$
A

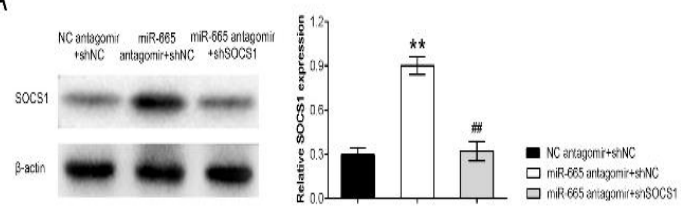

B
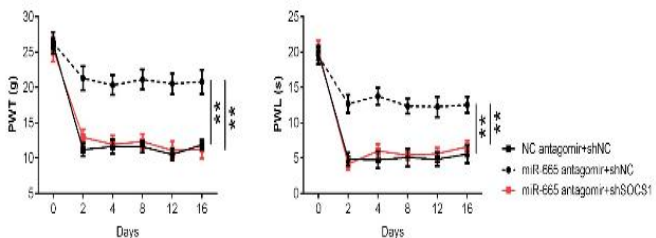

C
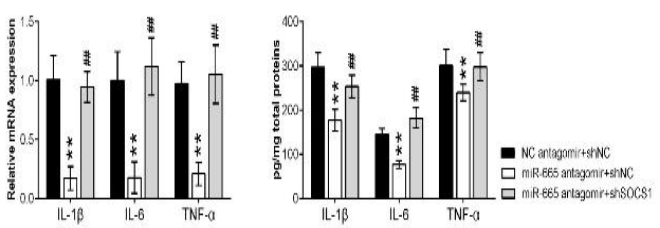

Figure 5: miR-665 aggravated neuropathic pain by regulating SOCS1 expression. A. The protein levels of SOCS1 in $\mathrm{CCl}$ rats injected with miR-665 antagomir and adenovirus plasmid of shSOCS1 to the medullary sheath were determined using Western blot. B. The PWT and PWL were measured in $\mathrm{CCl}$ rats injected with miR-665 antagomir and adenovirus plasmid of shSOCS1. C. Levels of IL-1 $\beta$, IL-6, and TNF- $\alpha$ in CCI rats injected with miR-665 antagomir and adenovirus plasmid of shSOCS1 as determined using qRT-PCR assay and ELISA assay; ${ }^{* *} p<0.01, \# \# p<0.01$

In the present study, the significant differences of mechanical and thermal hyperalgesia in $\mathrm{CCl}$ and sham rats indicated successful establishment of the $\mathrm{CCl}$ model. miR-665 was clearly elevated in the spinal cord and microglia in $\mathrm{CCl}$ rats. These results are consistent with the study reported by $\mathrm{Li}$ et al [9] in which miR-665 was overexpressed in active colitis. Furthermore, highly expressed miR-665 was observed in intestinal ischemia/reperfusion [18], gastric adenocarcinoma tissues and cells [19], and endothelial cells in heart failure [20]. Similarly, miR-665 was overexpressed in the dorsal root ganglion of $\mathrm{CCl}$ rats.

Abnormally expressed miR-665 in $\mathrm{CCl}$ rats implied that miR-665 might regulate neuropathic pain. Therefore, miR-665 antagomir was used to decrease the level of miR-665 in the spinal cord of rats. The decreased PWT and PWL caused by $\mathrm{CCl}$ were increased by miR-665 antagomir. Thus, the down-regulation of miR-665 alleviated the pain sensitivity of $\mathrm{CCl}$ rats. Neuropathic pain is usually accompanied by an inflammatory response, which is a vital pathogenic mechanism underlying neuropathic pain [21]. Thus, the influence of miR-665 on the production of 
inflammation factors such as IL-1 $\beta$, IL-6, and TNF- $\alpha$ was studied. The levels of IL-1 $\beta, I L-6$, and TNF- $\alpha$ were increased in the $\mathrm{CCl}$ model but decreased by miR-665 down-regulation. The down-regulation of miR-665 alleviated both pain sensitivity and the inflammation response of neuropathic pain. Thus, miR-665 may be a promising therapeutic target for neuropathic pain.

Because miRNAs usually modulate disease development by altering the expression of target genes, the potential target genes of miR-665 were screened. Results revealed that SOCS1 was the target gene of miR-665, and SOCS1 was reversely regulated by miR-665. Previous studies showed that SOCS1 is a negative modulator of cytokine signaling and involved in the inflammation response, cell proliferation, cell differentiation, and cell metastasis [22,23]. Moreover, SOCS1 was down-regulated in the spinal cord of $\mathrm{CCl}$ rats. These results are in agreement with findings in previous studies $[24,25]$. Zhang et al showed SOCS1 is downregulated in spinal neurons in $\mathrm{CCl}$ mice and is involved in neuropathic pain regulation [24]. SOCS1 overexpression abolishes the CClinduced behavioral pain, proinflammatory cytokine production, and activation of neurons, astrocytes, and microglia [24]. In addition, a separate study found miR-221 alleviates neuropathic pain via targeting SOCS1 [26].

miR-19a also modulates neuropathic pain progression via targeting SOCS1. Hence, miR665 may regulate neuropathic pain by targeting SOCS1. To investigate whether miR-665 regulates neuropathic pain through regulating SOCS1, the expressions of miR-665 and SOCS1 were changed concurrently in $\mathrm{CCl}$ rats. Furthermore, down-regulation of SOCS1 reversed the changes in PWT, PWL, and inflammatory factors induced by down-regulation of miR-665. Based on the above evidence, miR665 aggravated neuropathic pain via targeting SOCS1.

\section{CONCLUSION}

The findings of this study show that downregulation of miR-665 alleviates neuropathic pain by targeting SOCS1. Thus, miR-665 is a potential therapeutic target for neuropathic pain.

\section{DECLARATIONS}

\section{Conflict of interest}

No conflict of interest is associated with this work.

\section{Contribution of authors}

We declare that this work was done by the authors named in this article and all liabilities pertaining to claims relating to the content of this article will be borne by the authors. Yongqiang Lin and Mengjia Li designed the study, supervised the data collection, analyzed the data, Gaofeng Rao interpreted the data and prepared the manuscript for publication; Wenfu Zhang and Xuyan Chen supervised the data collection, analyzed the data, and reviewed the draft of the manuscript. All authors read and approved the manuscript.

\section{Open Access}

This is an Open Access article that uses a funding model which does not charge readers or their institutions for access and distributed under the terms of the Creative Commons Attribution License (http://creativecommons.org/licenses/by/ 4.0) and the Budapest Open Access Initiative (http://www.budapestopenaccessinitiative.org/rea d), which permit unrestricted use, distribution, and reproduction in any medium, provided the original work is properly credited.

\section{REFERENCES}

1. Colloca L, Ludman T, Bouhassira D, Baron R, Dickenson $A H$, Yarnitsky D, Freeman $R$, Truini A, Attal N, Finnerup NB. Neuropathic pain. Nat Rev Dis Primers 2017; 3: 17002.

2. Dworkin RH, O'Connor AB, Backonja $M$, Farrar JT, Finnerup NB, Jensen TS, Kalso EA, Loeser JD, Miaskowski C, Nurmikko $T J$ et al. Pharmacologic management of neuropathic pain: evidence-based recommendations. Pain 2007; 132(3): 237-251.

3. Murnion BP. Neuropathic pain: current definition and review of drug treatment. Aust Prescr 2018; 41(3): 6063.

4. Bartel DP. MicroRNAs: target recognition and regulatory functions. Cell 2009; 136(2): 215-233.

5. Hu Y, Liu Q, Zhang M, Yan Y, Yu H, Ge L. MicroRNA362-3p attenuates motor deficit following spinal cord injury via targeting paired box gene 2. J Integr Neurosci 2019; 18(1): 57-64.

6. Sun $W$, Zhang $L$, Li R. Overexpression of miR-206 ameliorates chronic constriction injury-induced neuropathic pain in rats via the MEKJERK pathway by targeting brain-derived neurotrophic factor. Neurosci Lett 2017; 646: 68-74.

7. Cai W, Zhang Y, Liu Y, Liu H, Zhang Z, Su Z. Effects of miR-150 on neuropathic pain process via targeting AKT3. Biochem Biophys Res Commun 2019; 517(3): 532-537. 
8. Zhang X, Guo $H$, Xie A, Liao O, Ju F, Zhou Y. MicroRNA-144 relieves chronic constriction injury-induced neuropathic pain via targeting RASA1. Biotechnol Appl Biochem 2020; 67(2): 294-302.

9. Li M, Zhang S, Qiu Y, He Y, Chen B, Mao R, Cui Y, Zeng $Z$, Chen M. Upregulation of miR-665 promotes apoptosis and colitis in inflammatory bowel disease by repressing the endoplasmic reticulum stress components XBP1 and ORMDL3. Cell Death Dis 2017; 8(3): e2699.

10. Lu X, Lv S, Mi Y, Wang L, Wang G. Neuroprotective effect of miR-665 against sevoflurane anesthesiainduced cognitive dysfunction in rats through PI3K/Akt signaling pathway by targeting insulin-like growth factor 2. Am J Transl Res 2017; 9(3): 1344-1356.

11. Zhang J, Zhang H, Zi T. Overexpression of microRNA141 relieves chronic constriction injury-induced neuropathic pain via targeting high-mobility group box 1 . Int J Mol Med 2015; 36(5): 1433-1439.

12. Care IoLARCo, Animals UoL: Guide for the care and use of laboratory animals: US Department of Health and Human Services, Public Health Service, National; 1986.

13. Inoue $N$, Ito $S$, Tajima $K$, Nogawa $M$, Takahashi $Y$, Sasagawa T, Nakamura A, Kyoi T. Etodolac attenuates mechanical allodynia in a mouse model of neuropathic pain. J Pharmacol Sci 2009: 0904030223-0904030223.

14. Zhou TE, Rivera JC, Bhosle VK, Lahaie I, Shao Z, Tahiri H, Zhu T, Polosa A, Dorfman A, Beaudry-Richard A. Choroidal involution is associated with a progressive degeneration of the outer retinal function in a model of

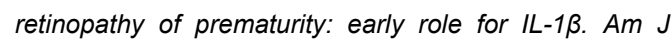
Pathol 2016; 186(12): 3100-3116.

15. Wu D, Cheng Y-g, Huang X, Zhong M-w, Liu S-z, Hu S-y. Downregulation of IncRNA MALAT1 contributes to renal functional improvement after duodenal-jejunal bypass in a diabetic rat model. J Physiol Biochem 2018; 74(3): 431-439.

16. Andersen HH, Duroux M, Gazerani P. MicroRNAs as modulators and biomarkers of inflammatory and neuropathic pain conditions. Neurobiol Dis 2014; 71 : 159-168.
17. Wu FX, Pan RR, Yu WF, Liu R. The Anti-Nociception Effect of Dezocine in a Rat Neuropathic Pain Model. Transl Perioper Pain Med 2014; 1(1): 5-8.

18. Li Z, Wang G, Feng $D$, Zu G, Li Y, Shi X, Zhao $Y$, Jing $H$, Ning $S$, Le $W$. Targeting the miR-665-3p-ATG4Bautophagy axis relieves inflammation and apoptosis in intestinal ischemia/reperfusion. Cell Death Dis 2018; 9(5): 483.

19. Tang H, Long Q, Zhuang K, Yan Y, Han K, Guo H, Lu X. miR-665 promotes the progression of gastric adenocarcinoma via elevating FAK activation through targeting SOCS3 and is negatively regulated by IncRNA MEG3. J Cell Physiol 2020; 235(5): 4709-4719.

20. Fan J, Li H, Nie X, Yin Z, Zhao Y, Zhang X, Yuan S, Li Y, Chen $C$, Wang DW. MiR-665 aggravates heart failure via suppressing CD34-mediated coronary microvessel angiogenesis. Aging (Albany NY) 2018; 10(9): 2459.

21. Jiang B-C, Sun $W-X$, He L-N, Cao D-L, Zhang Z-J, Gao $Y$-J. Identification of IncRNA expression profile in the spinal cord of mice following spinal nerve ligationinduced neuropathic pain. Mol Pain 2015; 11(1): 43.

22. Sun $K$, Zhang G. Long noncoding RNA CASC2 suppresses esophageal squamous cell carcinoma progression by increasing SOCS1 expression. Cell Biosci. 2019; 9(1): 1-14.

23. Xi W, Zhao X, Wu M, Fu X, Jia W, Lu M, Li H. MiR-214 promotes renal fibrosis in diabetic nephropathy via targeting SOCS1. Trop J Pharm Res 2019; 18(5).

24. Zhang $T$, Sun $K$, Shen $W$, Qi L, Yin W, Wang $L W$. SOCS1 regulates neuropathic pain by inhibiting neuronal sensitization and glial activation in mouse spinal cord. Brain Res Bull 2016; 124: 231-237.

25. Tan Y, Yang J, Xiang K, Tan Q, Guo Q. Suppression of microRNA-155 attenuates neuropathic pain by regulating SOCS1 signalling pathway. Neurochem Res 2015; 40(3): 550-560.

26. Xia L, Zhang Y, Dong T. Inhibition of microRNA-221 alleviates neuropathic pain through targeting suppressor of cytokine signaling 1 . Journal of Molecular Neuroscience 2016; 59(3): 411-420. 